\title{
Synthesis and Crystal Structure of a Meso-Meso Directly Linked Bisporphyrin
}

Mathias O. Senge, ${ }^{\mathrm{a}, \mathrm{b}}$ Monica Pintea, ${ }^{\mathrm{a}}$ and Aoife A. Ryan ${ }^{\mathrm{a}}$

${ }^{\text {a }}$ School of Chemistry, SFI Tetrapyrrole Laboratory, Trinity College Dublin, Dublin 2, Ireland

${ }^{b}$ Medicinal Chemistry, Institute of Molecular Medicine, Trinity Centre for Health Sciences, Trinity College Dublin, St James's Hospital, Dublin 8, Ireland

Reprint requests to Prof. Dr. M. O. Senge. Fax: \#353-1-896-8537. E-mail: sengem@tcd.ie

Z. Naturforsch. 2011, 66b, XXXX-XXXX; received March 30, 2011

We report the synthesis and characterization of a meso-meso directly linked bisporphyrin 6 bearing both alkyl and aryl residues. Oxidative fusing results in a mesomeso, $\beta-\beta, \beta-\beta$-linked bisporphyrin. The first crystal structure analysis of a meso-meso directly linked porphyrin dimer $\mathbf{6}$ shows the inequivalency of the two porphyrin units with regard to the macrocycle conformation. Quite distinct mixings of distortion modes were observed for the two aromatic macrocycles.

Key words: Porphyrins, Conformational Analysis, Bisporphyrins, Tetrapyrroles, Crystal Structure 
Directly-linked oligoporphyrins $\mathbf{1}$ have attracted considerable interest in recent years $[1,2]$. This is mainly related to the preparation of polymers and of the ability of the meso-meso-linked dimers to be oxidatively fused to yield meso-meso, and $2 \times \beta-\beta$-fused systems 2 (linked in the 2, 5, and 7 position) [3]. Synthetically the meso-meso linked bisporphyrins $1(n=1)$ can be prepared either through treatment of porphyrins with free meso positions with $\mathrm{AgPF}_{6}$ [3a], total synthesis [4], $\mathrm{AgPF}_{6}+\mathrm{I}_{2}$ [5], electrochemically [6] or Ullmann homocoupling [7]. Our approach to these systems involved the reaction of a precursor porphyrin with $\mathrm{RLi}$, in situ oxidation of the anion to the radical anion, followed by radial dimerization [8].

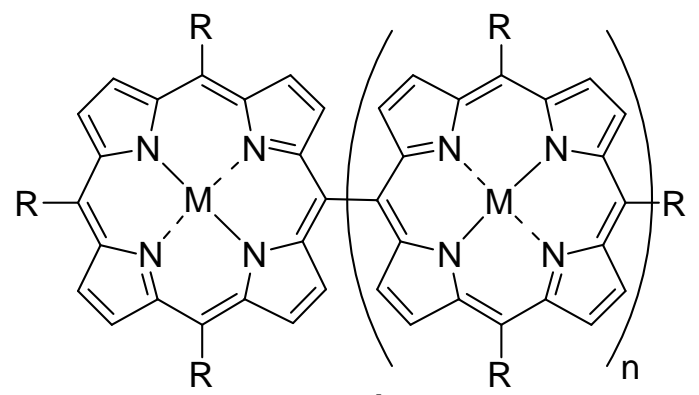

1

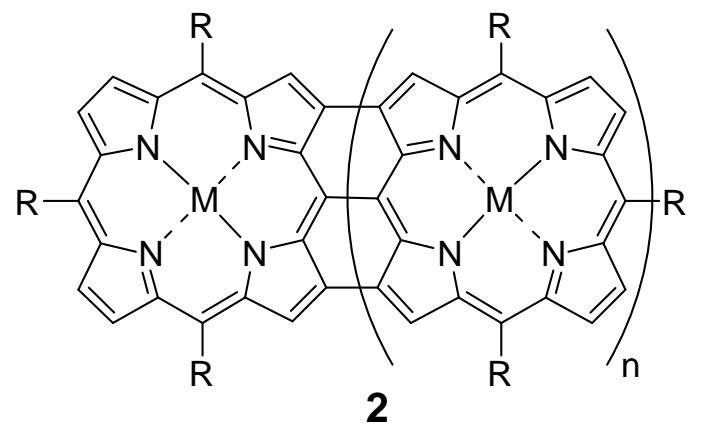

Surprisingly, despite the ongoing interest in these systems, only a few crystal structures of meso-meso-linked trisporphyrins have been reported [2b,9]. This is related to the low solubility of some of these compounds. Likewise, only limited studies on the preparation of unsymmetrical systems thereof or porphyrin arrays amenable to further 
functionalization have been published. In the context of our interest in the preparation of near-IR absorbers for applications in photodynamic therapy (PDT) [10] we have begun to explore the chemistry of these compounds with the goal in mind to make unsymmetrical and amphiphilic systems for use in preclinical studies [11-13].

Scheme 1 outlines our initial syntheses. In line with earlier results 5,15diphenylporphyrin [14] was treated with 6 equiv. of hexyl lithium followed by direct addition of DDQ without hydrolysis step and gave the meso-meso-linked dimer $\mathbf{6}$ as the main product in $40 \%$ yield. Formation of this compound was accompanied by small amounts of the mono- 4 [15] and dihexylated 5 side products. The dimer 6 could easily be converted through standard reactions into the zinc(II) complex 7. The UV/Vis spectrum of this dimer exhibits the characteristic split Soret band (422 and $455 \mathrm{~nm}$ ) and Q-bands at 568 and $608 \mathrm{~nm}$ in $\mathrm{CH}_{2} \mathrm{Cl}_{2}$. As shown by the crystal structure of the free base (vide supra) the two porphyrin rings lie almost orthogonal to each other, which results in a weak ground state interaction between the two chromophores [16]. 

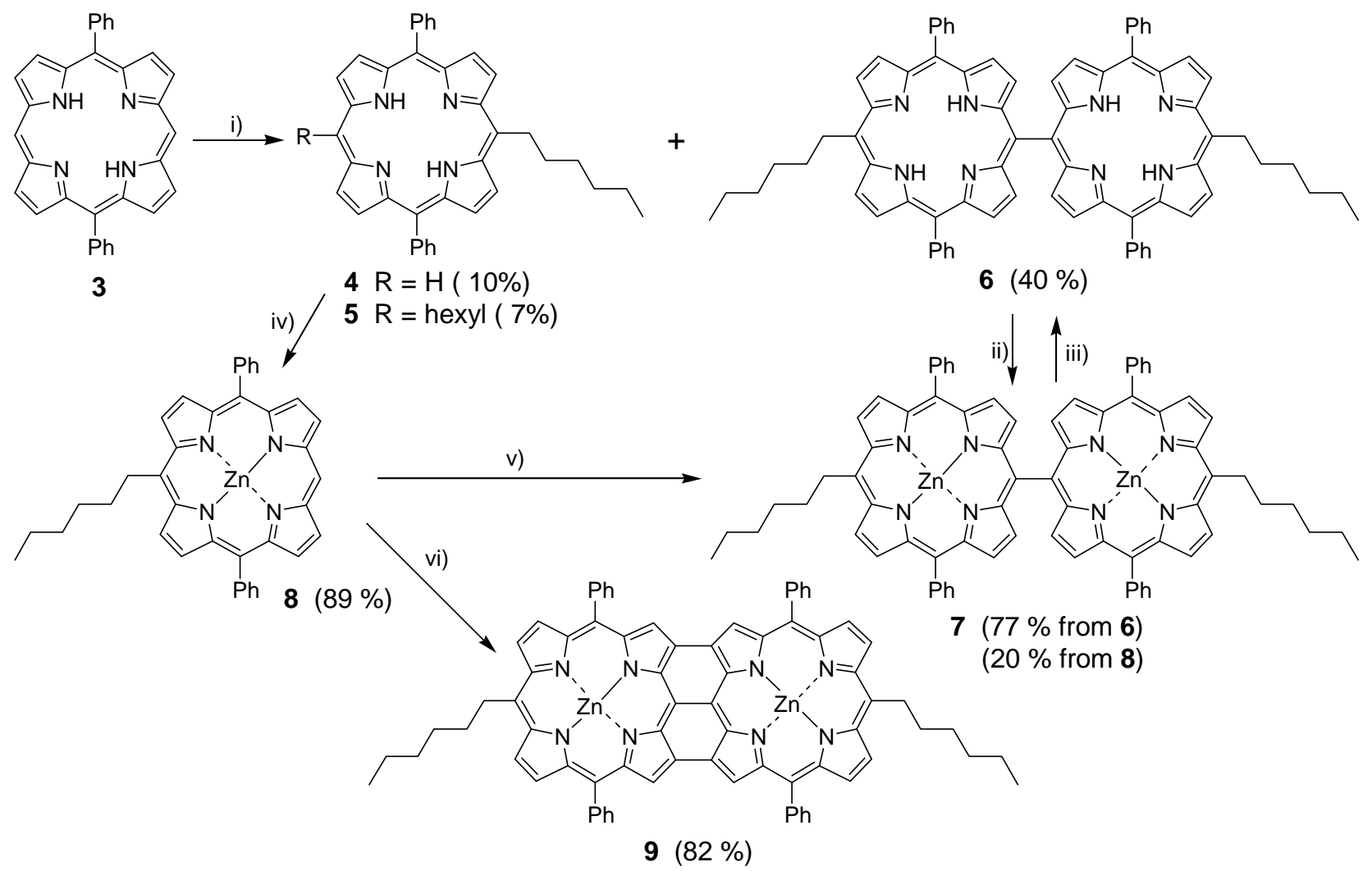

Scheme 1. Synthesis of meso-meso-linked bisporphyrins. i) $n \mathrm{HexLi}$, DDQ, THF $-78{ }^{\circ} \mathrm{C}$; ii) $\mathrm{Zn}(\mathrm{II})(\mathrm{OAc})_{2}, \mathrm{CH}_{3} \mathrm{OH}, \mathrm{CH}_{2} \mathrm{Cl}_{2}, 20^{\circ} \mathrm{C}$; iii) $\mathrm{Sc}(\mathrm{OTf})_{3}$, DDQ, toluene, $\Delta$; iv) $\mathrm{ZnO}$, TFA, $\left.\mathrm{CH}_{2} \mathrm{Cl}_{2} ; \mathrm{v}\right) \mathrm{Sc}(\mathrm{OTf})_{3}$, DDQ, toluene, $\Delta$; vi) $\mathrm{Sc}(\mathrm{OTf})_{3}$, DDQ, toluene, $20^{\circ} \mathrm{C}$.

Next we attempted the preparation of the respective triply-linked dimer 9. Reaction with 5 equiv. of DDQ and 5 equiv. of $\mathrm{Sc}(\mathrm{OTf})_{3}$ at $50{ }^{\circ} \mathrm{C}$ gave no fused product, and raising the temperature resulted in the formation of small amounts of the demetallated compound 6. Similarly, an increase of $\mathrm{Sc}(\mathrm{OTf})_{3}$ to 7 equiv. and heating to reflux resulted only in the formation of the singly-linked free base 7 . As an alternative route we prepared the zinc(II) complex 8 from 4 . Reaction with 5 equiv. of $\mathrm{Sc}(\mathrm{OTf})_{3}$ and 5 equiv. DDQ in toluene at $50{ }^{\circ} \mathrm{C}$ gave only the meso-meso-linked dimer 7 in $20 \%$ yield. Surprisingly, performing the same reaction at room temperature for $18 \mathrm{~h}$ resulted in the formation of the desired triply-linked bisporphyrin $\mathbf{9}$ in very good yield. Although identification proved to be difficult due to solubility and stability issues, its formation could clearly be 
proven by mass spectrometry and UV/Vis spectroscopy. The absorption spectrum is very similar to those of related systems in the literature [17] and exhibits two strong Soret-like bands at 426 and $576 \mathrm{~nm}$ and a long wavelength absorption at $1135 \mathrm{~nm}$ in THF.

For compound $\mathbf{6}$ we were able to grow crystals suitable for X-ray crystallographic structure analysis. The structure is shown in Fig. 1 and presents the first structure of a meso-meso directly linked bisporphyrin [9a]. The meso-meso single bond linkage is clearly indicated by the $\mathrm{C} 5-\mathrm{C} 25$ bond length of 1.503(3) $\AA$. The two tetrapyrrole rings are twisted against each other by $69.1(2)^{\circ}$. This value is similar to that found for the phenyl twist angle in various meso-tetraarylporphyrins [18]. The dihedral twist is clearly illustrated in the packing diagram shown in Fig. 2. Note, that the twist angle is different from the one in a related meso- $\beta$-linked porphyrin, 2-(5,10,15-triphenylporphyrin-20-yl)5,10,15,20-tetraphenylporphyrin, where an angle of $106^{\circ}$ was observed [19].

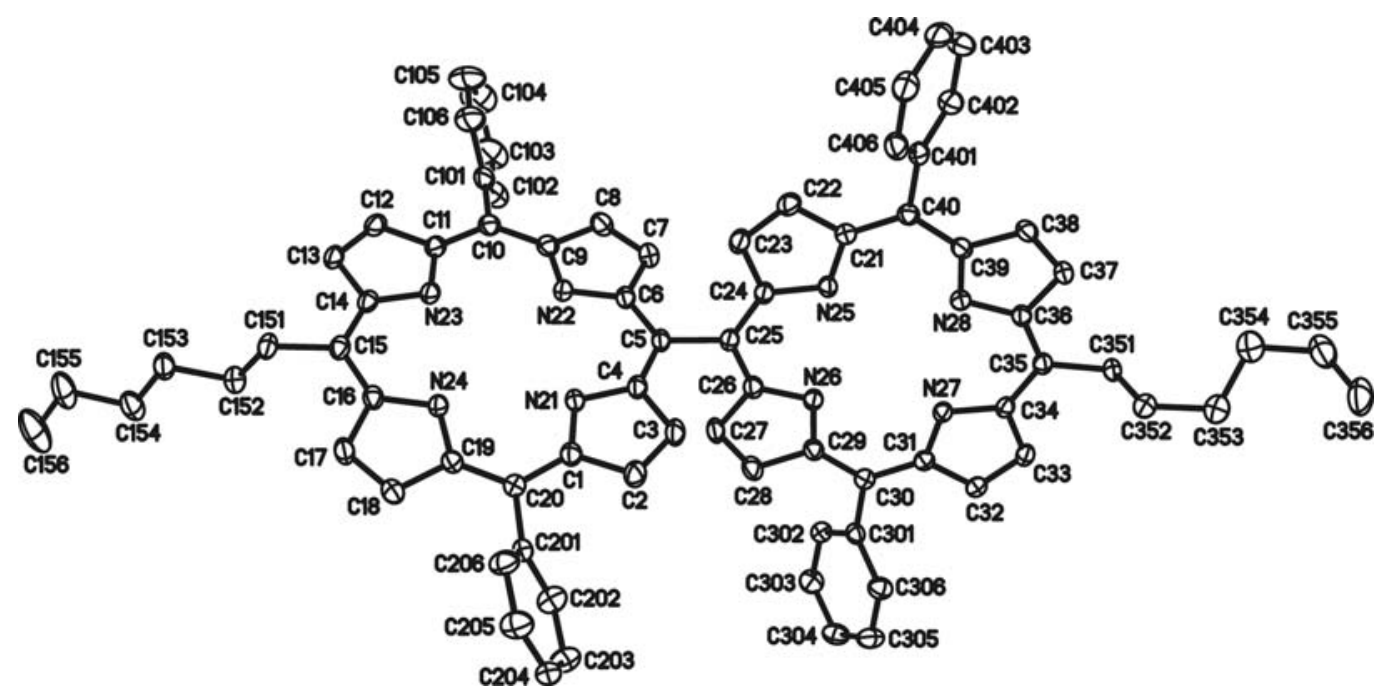

Fig. 1. View of the molecular structure of 6 in the crystal. Displacement ellipsoids are drawn at the $50 \%$ probability level. Hydrogen atoms have been omitted for clarity. 
The compound crystallized as a dichloromethane solvate (Fig. 2). Interestingly the compound does not crystallize with an inversion center. The first obvious difference is the conformation of the two hexyl side chains, one of which shows a more folded arrangement. The packing of the molecules is characterized by very weak $\pi-\pi$ interactions between neighboring ring II units. The mean plane separation is $3.34 \AA$, with a slip angle of $47.3^{\circ}$ and a center-to-center separation of $4.924 \AA$. The methylene chlorides of solvation are located in the voids between these units. The shortest intermolecular contact is $\mathrm{C} 11 \mathrm{~S} \cdots \mathrm{H} 405(2.903 \AA)$.

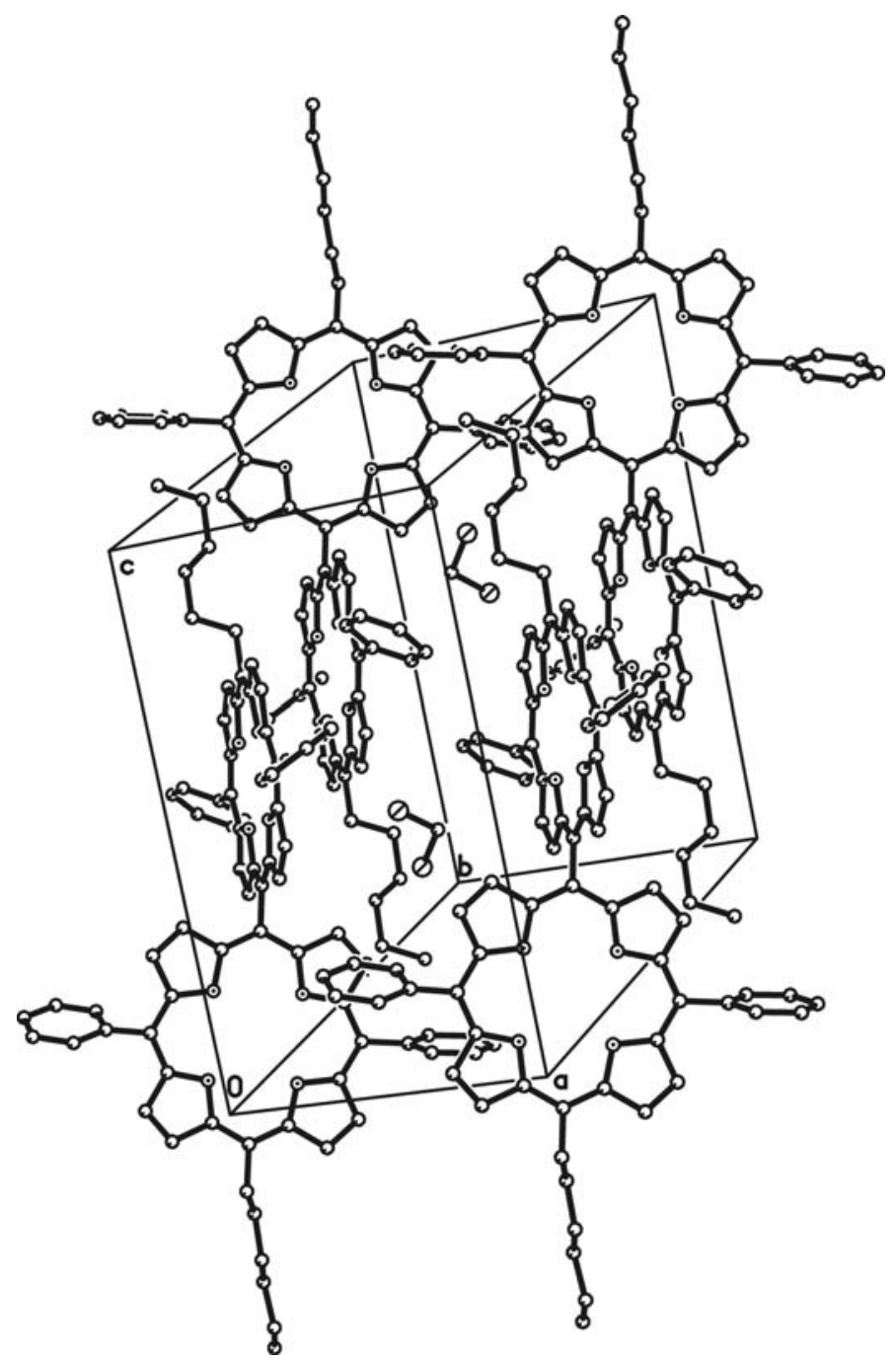


Fig. 2. View of the crystal structure of $\mathbf{6}$. Hydrogen atoms have been omitted for clarity.

More striking differences between the two porphyrin units were found upon an analysis of their conformation. The average deviation from planarity, i. e. $\Delta 24$, was found to be $0.094 \AA$ for macrocycle 1 and $0.047 \AA$ for macrocycle 2 . A more detailed inspection using a normal structural decomposition (NDS) [20] analysis revealed significant differences in the distortion modes and types of the two porphyrin macrocycles. Porphyrin unit 1 has about equal contributions from sad and ruf out-of-plane distortion and also exhibits $\operatorname{wav}(x)$ distortion. In contrast, porphyrin unit 2 has smaller contributions from these distortion types but has a significant wav(y) contribution. With regard to the in-plane distortions the main differences are larger $B 2 g$ contributions in unit 2 and larger A2g contributions in unit 1 (Fig. 3). Such large difference in the type and composition of the distortion modes are rare in bisporphyrins and implicate an interplay of packing forces and the mix of aryl- and alkyl-substituent types [21].

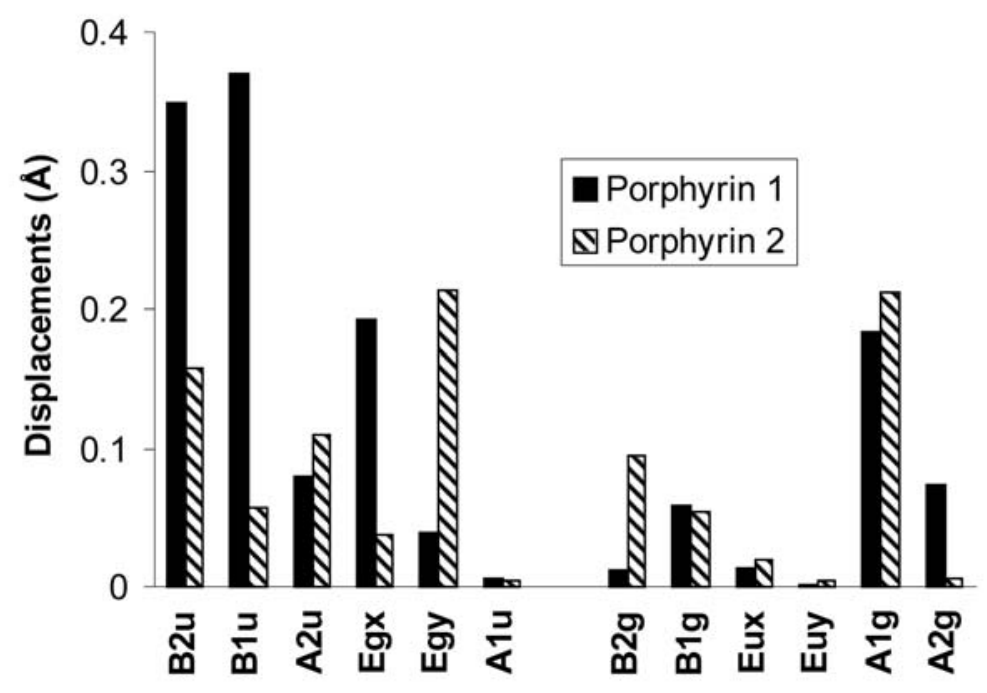


Fig. 3. Graphical representation of the displacements along the lowest-frequency coordinates that best simulate the two porphyrin units in molecule 6 . Signs have been omitted for ease of comparison.

\section{Experimental}

For general conditions and instrumentation see reference [22].

\section{5,15-Dihexyl-10,20-diphenylporphyrin (5)}

5,15-Diphenylporphyrin 3 (150 mg, $0.324 \mathrm{mmol}$ ) was dissolved in $80 \mathrm{~mL}$ of dry THF under an argon atmosphere and the reaction mixture was cooled to $-78^{\circ} \mathrm{C} . n$ - $\mathrm{HexLi}$ (1.4 mL, $3.245 \mathrm{mmol}$ ) was added dropwise over 15 minutes via syringe. The cold bath was removed and stirring continued 1.5 hours at room temperature. Next $0.5 \mathrm{~mL}$ of $\mathrm{H}_{2} \mathrm{O}$ was added and stirring continued for $15 \mathrm{~min}$. Then DDQ (730 mg, $3.245 \mathrm{mmol})$ in $10 \mathrm{~mL}$ of THF was added and the reaction mixture stirred for an additional hour. The reaction mixture was filtered through silica gel, followed by evaporation of the solvent. The crude reaction mixture was purified by column chromatography $(n$-hexane $:$ ethyl acetate $=100$ $: 1, \mathrm{v} / \mathrm{v})$. The first fraction was 5,15-dihexyl-10,20-diphenylporphyrin 5 (14 mg, 0.022 mmol, $7 \%$ as purple crystals, the second fraction contained 5-hexyl-10,20diphenylporphyrin 4 (17 $\mathrm{mg}, 0.031 \mathrm{mmol}, 10 \%)$ [15] as purple crystals, and the third fraction the directly linked dimer 6 (70 $\mathrm{mg}, 0.064 \mathrm{mmol}, 40 \%$ ) as purple crystals. M. p. > $300{ }^{\circ} \mathrm{C} .-R_{\mathrm{f}}=0.5(n$-hexane $:$ ethyl acetate $=1: 1, \mathrm{v} / \mathrm{v}) .-\mathrm{UV} / \mathrm{Vis}\left(\mathrm{CH}_{2} \mathrm{Cl}_{2}\right): \lambda_{\max }(\log \varepsilon)=$

419 (5.71), 518 (4.31), 554 (4.09), 598 (3.86), 656 nm (3.93). - ${ }^{1} \mathrm{H}$ NMR (400 MHz, $\left.\mathrm{CDCl}_{3}\right): \delta=-2.66(\mathrm{~s}, 2 \mathrm{H}, \mathrm{N} H), 0.95\left(\mathrm{t}, J=7.01, \mathrm{~Hz}, 3 \mathrm{H}, \mathrm{CH}_{2} \mathrm{CH}_{2} \mathrm{CH}_{2} \mathrm{CH}_{2} \mathrm{CH}_{2} \mathrm{CH}_{3}\right), 1.41$ (m, $2 \mathrm{H}, \mathrm{CH}_{2} \mathrm{CH}_{2} \mathrm{CH}_{2} \mathrm{CH}_{2} \mathrm{CH}_{2} \mathrm{CH}_{3}$ ), 1.51 (m, 2H, $\mathrm{CH}_{2} \mathrm{CH}_{2} \mathrm{CH}_{2} \mathrm{CH}_{2} \mathrm{CH}_{2} \mathrm{CH}_{3}$ ), 1.82 (m, 
$\left.2 \mathrm{H}, \mathrm{CH}_{2} \mathrm{CH}_{2} \mathrm{CH}_{2} \mathrm{CH}_{2} \mathrm{CH}_{2} \mathrm{CH}_{3}\right), 2.51\left(\mathrm{~m}, 2 \mathrm{H}, \mathrm{CH}_{2} \mathrm{CH}_{2} \mathrm{CH}_{2} \mathrm{CH}_{2} \mathrm{CH}_{2} \mathrm{CH}_{3}\right), 4.97$ (t, $J=7.16$ $\mathrm{Hz}, 2 \mathrm{H}, \mathrm{CH}_{2} \mathrm{CH}_{2} \mathrm{CH}_{2} \mathrm{CH}_{2} \mathrm{CH}_{2} \mathrm{CH}_{3}$ ), 7.81 (s, 6H, Ar- $H$ ), 8.20 (s, 4H, Ar- $H$ ), 8.89 (d, $J=$ $\left.5.26 \mathrm{~Hz}, 4 \mathrm{H}, H_{\beta}\right), 9.46\left(\mathrm{~d}, J=4.68 \mathrm{~Hz}, 2 \mathrm{H}, H_{\beta}\right), 9.62 \mathrm{ppm}\left(\mathrm{d}, J=4.68 \mathrm{~Hz}, 2 \mathrm{H}, H_{\beta}\right) .-{ }^{13} \mathrm{C}$ $\operatorname{NMR}\left(150 \mathrm{MHz}, \mathrm{CDCl}_{3}\right): \delta=13.9,22.5,29.5,30.0,31.7,35.1,35.4,38.5,38.6,111.1$, $112.6,119.9,121.1,121.2,126.3,126.4,126.5,126.6,126.7,127.6,127.8,134.3,134.5$, 141.1, 141.8 ppm. - HRMS ((+)-ES): $m / z$ calcd. for $\left[\mathrm{C}_{44} \mathrm{H}_{47} \mathrm{~N}_{4}\right][\mathrm{M}+\mathrm{H}]^{+}:$: 631.3808; found 631.3801 .

Bis(5-hexyl-10,20-diphenylporphyrin-15-yl) (6)

The compound was obtained from the reaction described for 5 as purple cyrstals (70 mg, 0.064 mmol, $40 \%)$. M. p. $>300{ }^{\circ} \mathrm{C} .-R_{\mathrm{f}}=0.37(n$-hexane $:$ ethyl acetate $=1: 1, \mathrm{v} / \mathrm{v})$. - UV/Vis $\left(\mathrm{CH}_{2} \mathrm{Cl}_{2}\right): \lambda_{\max }(\log \varepsilon)=418$ (5.28), 448 (5.33), 523 (4.64), 558 (4.13), $598 \mathrm{~nm}$ (4.12). - ${ }^{1} \mathrm{H}$ NMR (400 MHz, $\left.\mathrm{CDCl}_{3}, \mathrm{TMS}\right): \delta=-2.15$ (s, 4H, NH), 0.98 (t, $J=7.6, \mathrm{~Hz}$, $\left.6 \mathrm{H}, \mathrm{CH}_{2} \mathrm{CH}_{2} \mathrm{CH}_{2} \mathrm{CH}_{2} \mathrm{CH}_{2} \mathrm{CH}_{3}\right), 1.44\left(\mathrm{~m}, 4 \mathrm{H}, \mathrm{CH}_{2} \mathrm{CH}_{2} \mathrm{CH}_{2} \mathrm{CH}_{2} \mathrm{CH}_{2} \mathrm{CH}_{3}\right), 1.53(\mathrm{~m}, 4 \mathrm{H}$, $\left.\mathrm{CH}_{2} \mathrm{CH}_{2} \mathrm{CH}_{2} \mathrm{CH}_{2} \mathrm{CH}_{2} \mathrm{CH}_{3}\right), 1.88$ (m, 4H, $\left.\mathrm{CH}_{2} \mathrm{CH}_{2} \mathrm{CH}_{2} \mathrm{CH}_{2} \mathrm{CH}_{2} \mathrm{CH}_{3}\right), 2.63$ (m, 2H, $\mathrm{CH}_{2} \mathrm{CH}_{2} \mathrm{CH}_{2} \mathrm{CH}_{2} \mathrm{CH}_{2} \mathrm{CH}_{3}$ ), 5.14 (t, $J=7.16 \mathrm{~Hz}, 4 \mathrm{H}, \mathrm{CH}_{2} \mathrm{CH}_{2} \mathrm{CH}_{2} \mathrm{CH}_{2} \mathrm{CH}_{2} \mathrm{CH}_{3}$ ), 7.70 (s, 12H, Ar- $H), 8.05\left(\mathrm{~d}, J=3.51,4 \mathrm{H}, H_{\beta}\right), 8.22(\mathrm{~s}, 8 \mathrm{H}, \operatorname{Ar}-H), 8.55\left(\mathrm{~d}, J=4.68 \mathrm{~Hz}, 4 \mathrm{H}, H_{\beta}\right)$, $8.99\left(\mathrm{~d}, J=4.67 \mathrm{~Hz}, 4 \mathrm{H}, H_{\beta}\right), 9.60 \mathrm{ppm}\left(\mathrm{d}, J=5.26 \mathrm{~Hz}, 4 \mathrm{H}, H_{\beta}\right) .-{ }^{13} \mathrm{C} \mathrm{NMR}(150 \mathrm{MHz}$, $\left.\mathrm{CDCl}_{3}\right): \delta=13.7,22.6,29.2,30.1,31.8,35.5,38.8,52.8,53.0,53.4,53.6,116.5,120.3$, 121.8, 126.4, 127.4, 131.4, 134.2, 141.9 ppm. - HRMS ((+)-ES): m/z calcd. for $\left[\mathrm{C}_{76} \mathrm{H}_{67} \mathrm{~N}_{8}\right][\mathrm{M}+\mathrm{H}]^{+}:$1091.5472; found 1091.5489.

Bis\{(5-hexyl-10,20-diphenylporphyrin-15-ylato)zinc(II)\} (7) 
Bis(5-hexyl-10,20-diphenylporphyrin-15-yl) 6 (85 mg, $0.0788 \mathrm{mmol})$ and zinc acetate dihydrate $(205 \mathrm{mg}, 0.934 \mathrm{mmol})$ in $20 \mathrm{~mL}$ dried $\mathrm{CH}_{2} \mathrm{Cl}_{2}$ were reacted at room temperature for $3 \mathrm{~h}$. Purification of the product was carried out by recrystallization from $\mathrm{CH}_{2} \mathrm{Cl}_{2} / \mathrm{MeOH}$ to afford the desired compound (80 $\mathrm{mg}, 0.065 \mathrm{mmol}, 77 \%$ ) as purple crystals. M. p. $>300{ }^{\circ} \mathrm{C} .-R_{\mathrm{f}}=0.55\left(n\right.$-hexane $\left.: \mathrm{CH}_{2} \mathrm{Cl}_{2}=1: 2, \mathrm{v} / \mathrm{v}\right)$. $-\mathrm{UV} / \mathrm{Vis}\left(\mathrm{CH}_{2} \mathrm{Cl}_{2}\right)$ : $\lambda_{\max }(\log \varepsilon)=422$ (5.19), 456 (5.21), 568 (4.51), $608 \mathrm{~nm}(4.01) .-{ }^{1} \mathrm{H}$ NMR (400 MHz, $\mathrm{CDCl}_{3}$ ): $\delta=0.99$ (t, $J=7.60 \mathrm{~Hz}, 6 \mathrm{H}, \mathrm{CH}_{2} \mathrm{CH}_{2} \mathrm{CH}_{2} \mathrm{CH}_{2} \mathrm{CH}_{2} \mathrm{CH}_{3}$ ), 1.49 (m, 4H, $\mathrm{CH}_{2} \mathrm{CH}_{2} \mathrm{CH}_{2} \mathrm{CH}_{2} \mathrm{CH}_{2} \mathrm{CH}_{3}$ ); 1.63 (m, 4H, $\left.\mathrm{CH}_{2} \mathrm{CH}_{2} \mathrm{CH}_{2} \mathrm{CH}_{2} \mathrm{CH}_{2} \mathrm{CH}_{3}\right), 1.93$ (m, 4H, $\mathrm{CH}_{2} \mathrm{CH}_{2} \mathrm{CH}_{2} \mathrm{CH}_{2} \mathrm{CH}_{2} \mathrm{CH}_{3}$ ), 2.67 (m, 4H, $\mathrm{CH}_{2} \mathrm{CH}_{2} \mathrm{CH}_{2} \mathrm{CH}_{2} \mathrm{CH}_{2} \mathrm{CH}_{3}$ ), $5.16(\mathrm{t}, J=7.60 \mathrm{~Hz}$, $\left.4 \mathrm{H}, \mathrm{CH}_{2} \mathrm{CH}_{2} \mathrm{CH}_{2} \mathrm{CH}_{2} \mathrm{CH}_{2} \mathrm{CH}_{3}\right), 7.70(\mathrm{~s}, 12 \mathrm{H}, \mathrm{Ar}-H), 8.1\left(\mathrm{~d}, J=3.51 \mathrm{~Hz}, 4 \mathrm{H}, H_{\beta}\right), 8.25$ (m, 8H, Ar- $H), 8.64\left(\mathrm{~d}, J=3.51 \mathrm{~Hz}, 4 \mathrm{H}, H_{\beta}\right), 9.08\left(\mathrm{~d}, J=3.5 \mathrm{~Hz}, 4 \mathrm{H}, H_{\beta}\right), 9.69 \mathrm{ppm}(\mathrm{d}, J$ $\left.=4.1 \mathrm{~Hz}, 4 \mathrm{H}, H_{\beta}\right) .{ }^{13} \mathrm{C} \mathrm{NMR}\left(150 \mathrm{MHz}, \mathrm{CDCl}_{3}\right): \delta=13.7,22.3,29.2,29.9,31.5,38.6$, $120.8,125.9,126.9,128.6,131.4,131.6,133.2,133.8,142.3,148.9,149.6,154.5$ ppm; HRMS ((+)-LD): $m / z$ calcd. for $\left[\mathrm{C}_{76} \mathrm{H}_{62} \mathrm{~N}_{8} \mathrm{Zn}_{2}\right][\mathrm{M}]^{+}: 1214.3680$; found 1214.3636 .

Alternatively, this compound could be prepared in $20 \%$ yield via reaction of porphyrin 8 with 5 eq. of DDQ and 5 eq. of $\mathrm{Sc}(\mathrm{OTf})_{3}$ in dry toluene at $50{ }^{\circ} \mathrm{C}$ for 2 hours.

\section{(5-Hexyl-10,20-diphenylporphyrinato)zinc(II) (8)}

The free base porphyrin $5(90 \mathrm{mg}, 0.2 \mathrm{mmol})$ was dissolved in $20 \mathrm{~mL}$ of dried dichloromethane and treated with zinc oxide $(141.2 \mathrm{mg}, 1.2 \mathrm{mmol})$. After the addition of 4 drops of TFA, the reaction mixture turned green, and a color change back to red indicated completion of the reaction. The product was separated from $\mathrm{ZnO}$ and most of the TFA by filtration through a short silica column. Remaining traces of TFA were 
removed by washing with water. After drying with sodium sulfate, the solvent was removed in vacuo, and the product was purified by column chromatography (silica gel, dichloromethane). Purification of the product was carried out by recrystallization from $\mathrm{CH}_{2} \mathrm{Cl}_{2} / \mathrm{MeOH}$ and gave purple crystals (109 mg, $0.178 \mathrm{mmol}, 89 \%$ ). M. p. $>300{ }^{\circ} \mathrm{C}$. $R_{\mathrm{f}}=0.57\left(\mathrm{CH}_{2} \mathrm{Cl}_{2}: n\right.$-hexane $\left.=2: 1, \mathrm{v} / \mathrm{v}\right) .-\mathrm{UV} / \mathrm{Vis}\left(\mathrm{CH}_{2} \mathrm{Cl}_{2}\right): \lambda_{\max }(\log \varepsilon)=414(4.84)$, 452 (3.42), $543 \mathrm{~nm}(3.44) .-{ }^{1} \mathrm{H}$ NMR (400 MHz, $\left.\mathrm{CDCl}_{3}\right): \delta=0.95$ (t, $J=7.02 \mathrm{~Hz}, 3 \mathrm{H}$, $\mathrm{CH}_{2} \mathrm{CH}_{2} \mathrm{CH}_{2} \mathrm{CH}_{2} \mathrm{CH}_{2} \mathrm{CH}_{3}$ ), 1.44 (m, 2H, $\left.\mathrm{CH}_{2} \mathrm{CH}_{2} \mathrm{CH}_{2} \mathrm{CH}_{2} \mathrm{CH}_{2} \mathrm{CH}_{3}\right) 1.55$ (m, 2H, $\mathrm{CH}_{2} \mathrm{CH}_{2} \mathrm{CH}_{2} \mathrm{CH}_{2} \mathrm{CH}_{2} \mathrm{CH}_{3}$ ), 1.88 (m, 2H, $\left.\mathrm{CH}_{2} \mathrm{CH}_{2} \mathrm{CH}_{2} \mathrm{CH}_{2} \mathrm{CH}_{2} \mathrm{CH}_{3}\right), 2.62$ (m, 2H, $\mathrm{CH}_{2} \mathrm{CH}_{2} \mathrm{CH}_{2} \mathrm{CH}_{2} \mathrm{CH}_{2} \mathrm{CH}_{3}$ ), 5.13 (t, $J=7.16 \mathrm{~Hz}, 2 \mathrm{H}, \mathrm{CH}_{2} \mathrm{CH}_{2} \mathrm{CH}_{2} \mathrm{CH}_{2} \mathrm{CH}_{2} \mathrm{CH}_{3}$ ), 7.83 (s, 6H, Ar- $H), 8.26(\mathrm{~m}, 4 \mathrm{H}, \mathrm{Ar}-H), 9.07\left(\mathrm{~d}, J=5.26 \mathrm{~Hz}, 2 \mathrm{H}, H_{\beta}\right), 9.37$ (d, $J=4.68 \mathrm{~Hz}, 2 \mathrm{H}$, $\left.H_{\beta}\right), 9.66\left(\mathrm{~d}, J=4.68 \mathrm{~Hz}, 2 \mathrm{H}, H_{\beta}\right), 10.19 \mathrm{ppm}\left(\mathrm{s}, 1 \mathrm{H}, H_{\text {meso }}\right) .-{ }^{13} \mathrm{C} \mathrm{NMR}(100 \mathrm{MHz}$, $\left.\mathrm{CDCl}_{3}, \mathrm{TMS}\right): \delta=13.7,22.3,29.9,31.5,35.4,38.7,104.6,119.4,121.6,126.1,128.3$, 130.9, 131.6, 132.0, 133.8, 134.0, 142.3, 149.1, 149.2 ppm. - HRMS ((+)-ES): $m / z$ calcd. for $\left[\mathrm{C}_{38} \mathrm{H}_{33} \mathrm{~N}_{4} \mathrm{Zn}\right][\mathrm{M}+\mathrm{H}]^{+}$: 609.2006; found 609.1997.

Bis\{(5-hexyl-10,20-diphenylporphyrin-13,15,17-triylato)zinc(II)\} (9)

(5-Hexyl-10,20-diphenylporphyrinato)zinc(II) $\quad 8 \quad\left(\begin{array}{llllll}30.5 & \mathrm{mg}, & 0.05 & \mathrm{mmol}) & \text { was }\end{array}\right.$ dissolved in dry toluene (freshly distilled over $\mathrm{CaH}_{2}$ ). The flask was degassed with argon and heated to $50{ }^{\circ} \mathrm{C}$. Then DDQ $(56.75 \mathrm{mg}, 0.25 \mathrm{mmol})$ and $\mathrm{Sc}(\mathrm{OTf})_{3}(123.04 \mathrm{mg}, 0.25$ mmol) in dry toluene were added, and stirring continued for 3 hours. THF $(15 \mathrm{~mL})$ was added and the reaction mixture was stirred for 60 minutes. The reaction mixture was filtered through silica gel and the solvent removed under reduced pressure. The reaction mixture was purified by column chromatography $\left(\mathrm{CH}_{2} \mathrm{Cl}_{2}: \mathrm{CH}_{3} \mathrm{OH}=50: 1, \mathrm{v} / \mathrm{v}\right)$ and 
gave purple crystals $(25 \mathrm{mg}, 0.020 \mathrm{mmol}, 82 \%)$. M. p. $>300{ }^{\circ} \mathrm{C} .-R_{\mathrm{f}}=0.4\left(\mathrm{CH}_{2} \mathrm{Cl}_{2}\right.$ : $\left.\mathrm{CH}_{3} \mathrm{OH}=40: 1, \mathrm{v} / \mathrm{v}\right) .-\mathrm{UV} / \mathrm{Vis}(\mathrm{THF}): \lambda_{\max }(\log \varepsilon)=426$ (3.88), 479 (3.59), 576 (3.90), 995 (3.03), $1135 \mathrm{~nm}(3.30) .-{ }^{1} \mathrm{H} \mathrm{NMR}\left(600 \mathrm{MHz}, \mathrm{CDCl}_{3}\right): \delta=0.90$ (t, $J=13.41 \mathrm{~Hz}, 3 \mathrm{H}$, $\left.\mathrm{CH}_{2} \mathrm{CH}_{2} \mathrm{CH}_{2} \mathrm{CH}_{2} \mathrm{CH}_{2} \mathrm{CH}_{3}\right), \quad 1.58\left(\mathrm{~m}, 2 \mathrm{H}, \mathrm{CH}_{2} \mathrm{CH}_{2} \mathrm{CH}_{2} \mathrm{CH}_{2} \mathrm{CH}_{2} \mathrm{CH}_{3}\right) 1.84$ (m, 2H, $\left.\mathrm{CH}_{2} \mathrm{CH}_{2} \mathrm{CH}_{2} \mathrm{CH}_{2} \mathrm{CH}_{2} \mathrm{CH}_{3}\right), 1.98$ (m, 2H, $\left.\mathrm{CH}_{2} \mathrm{CH}_{2} \mathrm{CH}_{2} \mathrm{CH}_{2} \mathrm{CH}_{2} \mathrm{CH}_{3}\right), 2.07$ (m, 2H, $\mathrm{CH}_{2} \mathrm{CH}_{2} \mathrm{CH}_{2} \mathrm{CH}_{2} \mathrm{CH}_{2} \mathrm{CH}_{3}$ ), $3.98\left(\mathrm{t}, J=6.06 \mathrm{~Hz}, 2 \mathrm{H}, \mathrm{CH}_{2} \mathrm{CH}_{2} \mathrm{CH}_{2} \mathrm{CH}_{2} \mathrm{CH}_{2} \mathrm{CH}_{3}\right.$ ), 7.00 (s, 4H, $\left.H_{\beta}\right), 7.56(\mathrm{~m}, 20 \mathrm{H}, \operatorname{Ar}-H), 7.74\left(\mathrm{~m}, 4 \mathrm{H}, H_{\beta}\right), 8.23\left(\mathrm{~m}, 4 \mathrm{H}, H_{\beta}\right)$, ppm. - HRMS ((+)ES): $m / z$ calcd. for $\left[\mathrm{C}_{76} \mathrm{H}_{58} \mathrm{~N}_{8} \mathrm{Zn}_{2}\right][\mathrm{M}+\mathrm{H}]^{+}: 1210.3367$; found 1210.3315 .

X-Ray structure determination of (6)

Growth and handling of crystals followed the concept developed by Hope [23]. Intensity data for all compounds were collected with an Rigaku CCD system using graphite-monochromated $\mathrm{MoK}_{\alpha}$ radiation $(\lambda=0.71073 \AA)$. The intensities were corrected for Lorentz, polarization and absorption effects. Nonhydrogen atoms were refined with anisotropic thermal parameters, and hydrogen atoms were placed into geometrically calculated positions and refined using a ridging model. The structure was solved with Direct Methods using the SHELXTL PLUS program system and refined against $\left|F^{2}\right|$ with the program XL from SHELX-97 using all data [24].

Crystal data: $\mathrm{C}_{76} \mathrm{H}_{66} \mathrm{~N}_{8} \cdot \mathrm{CH}_{2} \mathrm{Cl}_{2}, M=1176.29$, triclinic, space group $P-1, a=$ 11.448(2), $b=15.175(2), c=18.459(2) \AA, \alpha=77.168(4)^{\circ}, \beta=86.858(5)^{\circ}, \gamma=$ 82.364(4) $)^{\circ}, V=3098.0(7) \AA^{3}, Z=2, \mathrm{~T}=98 \mathrm{~K}, \mu\left(\mathrm{MoK}_{\alpha}\right)=0.074 \mathrm{~cm}^{-1}, 23512$ reflections measured, 10810 unique reflections measured $\left(R_{\text {int }}=0.0208\right), 805$ parameters, 9615 reflections with $I>2.0 \sigma(I)$, refinement against $\left|\mathrm{F}^{2}\right|, R 1(I>2.0 \sigma(I))=0.0582, w R 2$ (all 
data) $=0.1521, S=1.034, \rho_{\max }=0.793$. Refinement: Hydrogen atoms at N25 and N27 were located in difference maps and refined using the standard riding model. For N21, N22, N23, N24 hydrogen atoms with $50 \%$ occupancy were added using the riding model. The methylene chloride of solvation is disordered, and the two $\mathrm{Cl}$ atoms were refined as disordered over two positions with free occupancy variables. Several atoms still show high librational movement.

CCDC 815159 (6) contains the supplementary crystallographic data for this paper. These data can be obtained free of charge from The Cambridge Crystallographic Data Centre via www.ccdc.cam.ac.uk/data_request/cif.

\section{Acknowledgement}

This work was supported by grants from Science Foundation Ireland (SFI P.I. 09/IN.1/B2650) and the Health Research Board (HRB Translational Research Award 2007 TRA/2007/11).

[1] a) A. Osuka, H. Shimidzu, Angew. Chem. 1997, 109, 93-95, Angew. Chem. Int. Ed. Engl. 1997, 36, 135-137; b) N. Aratani, A. Osuka, Y. H. Kim, D. H. Jeong, D. Kim, Angew. Chem. 2000, 112, 117-121, Angew. Chem. Int. Ed. 2000, 39, 1458-1462; c) N. Yoshida, N. Aratani, A. Osuka, Chem. Commun. 2000, 197198. 
[2] a) D. Kim, A. Osuka, Acc. Chem. Res. 2004, 37, 735-745; b) N. Aratani, A. Takagi, Y. Yanagawa, T. Matsumoto, T. Kawai, Z. S. Yoon, D. Kim, A. Osuka, Chem. Eur. J. 2005, 11, 3389-3404.

[3] a) A. Tsuda, H. Furuta, A. Osuka, Angew. Chem. 2000, 112, 2649-2652, Angew. Chem. Int. Ed. 2000, 39, 2549-2552; b) A. Tsuda, A. Osuka, Science 2001, 293, 79-82.

[4] R. G. Khoury, L. Jaquinod, K. M. Smith, Chem. Commun. 1997, 1057-1058.

[5] N. Yoshida, K. Shimidzu, A. Osuka, Chem. Lett. 1998, 55-56.

[6] T. Ogawa, Y. Nishimoto, N. Yoshida, N. Ono, A. Osuka, Chem. Commun. 1998, $337-338$.

[7] X. Q. Lu, Y. Guo, Q. Y. Chen, Synlett 2011, 77-80.

[8] a) M. O. Senge, X. D. Feng, Tetrahedron Lett. 1999, 40, 4165-4168; b) M. O. Senge, X. D. Feng, J. Chem. Soc., Perkin Trans. 1 2000, 3615-3621; c) M. O. Senge, Acc. Chem. Res. 2005, 38, 733-743.

[9] a) Cambride Structural Database, Version 5.32, release November 2010: F. H. Allen, Acta Cryst. 2002, B58, 380-388; b) N. Aratani, A. Osuka, Chem. Commun. 2008, 4067-4069; c) J. Song, N. Aratani, P. Kim, D. Kim, H. Shinokubo, A. Osuka, Angew. Chem. 2010, 122, 3699-3702, Angew. Chem. Int. Ed. 2010, 49, $3617-3620$.

[10] D. Kessel, Photochem. Photobiol. 1984, 39, 851-859.

[11] M. O. Senge, Chem. Commun. 2011, 47, 1943-1960.

[12] S. Ben-Dror, I. Bronshtein, A. Wiehe, B. Röder, M. O. Senge, B. Ehrenberg, Photochem. Photobiol. 2006, 82, 695-701. 
[13] M. B. Bakar, M. Oelgemöller, M. O. Senge, Tetrahedron 2009, 65, 7064-7078.

[14] R. W. Boyle, C. Brückner, J. Posakony, B. R. James, D. Dolphin, Org. Synth. 1999, 76, 287-293.

[15] a) A. Wiehe, Y. M. Shaker, J. C. Brandt, S. Mebs, M. O. Senge Tetrahedron 2005, 61, 5535-5564; b) M. O. Senge, unpublished results..

[16] N. Yoshida, T. Ishizuka, A. Osuka, D. H. Jeong, H. S. Cho, D. Kim, Y. Matsuzaki, A. Nogami, K. Tanaka, Chem. Eur. J. 2003, 9, 68-75.

[17] S. Hiroto, A. Osuka, J. Org. Chem. 2005, 70, 4054-4058.

[18] M. O. Senge in The Porphyrin Handbook, Eds.: K. M. Kadish, K. M. Smith, R. Guilard, Vol. 10, Academic Press, San Diego, 2000, pp. 1.

[19] M. O. Senge, B. Rößler, J. von Gersdorff, A. Schäfer, H. Kurreck, Tetrahedron Lett. 2004, 45, 3363-3367.

[20] a) L. Shelnutt in The Porphyrin Handbook, Eds.: K. M. Kadish, K. M. Smith, R. Guilard, Vol. 7, Academic Press, San Diego, 2000, pp. 167; b) Normal structural decomposition $\quad$ engine $\quad$ Version http://jasheln.unm.edu/jasheln/content/nsd/NSDengine/nsd index.htm, accessed $03 / 22 / 2011$.

[21] M. O. Senge, Chem. Commun. 2006, 243-256.

[22] M. Davis, M. O. Senge, O. B. Locos, Z. Naturforsch. 2010, 65b, 1472-1484.

[23] H. Hope, Prog. Inorg. Chem. 1994, 41, 1-19.

[24] G. M. Sheldrick, Acta Crystallogr. 2008, A64, 112-122. 
Figure for Content Section:

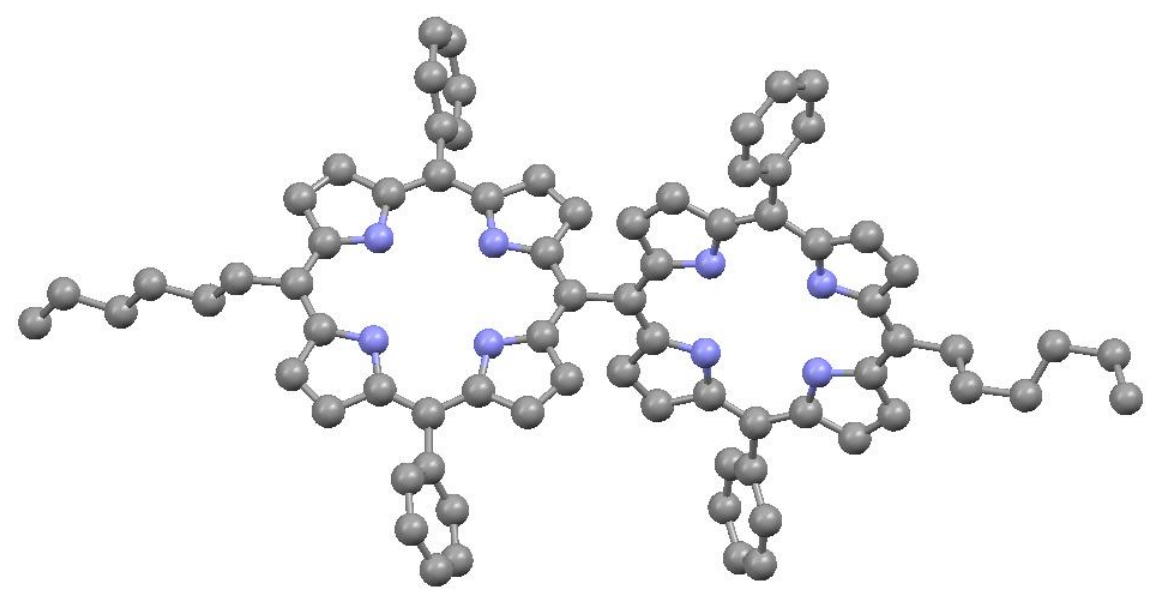

